\title{
ON MAPS: CONTINUOUS, CLOSED, PERFECT, AND WITH CLOSED GRAPH
}

\author{
G.L. GARG and ASHA GOEL \\ Department of Mathematics \\ Punjabi University \\ Patiala-147002, India
}

(Received January 11, 1994 and in revised form September 29, 1995)

\begin{abstract}
This paper gives relationships between continuous maps, closed maps, perfect maps, and maps with closed graph in certain classes of topological spaces.
\end{abstract}

KEY WORDS AND PHRASES. Continuous, closed, perfect, closed graph, B-W compact, Frechet, fiber, Hausdorff, regular, compact, countably compact.

1992 AMS SUBJECT CLASSIFICATION CODES.54C05,54C10,54D30.

\section{INTRODUCTION.}

Throughout, by a space we shall mean a topological space. No separation axioms are assumed and no map is assumed to be continuous or onto unless mentioned explicitly; $\operatorname{cl}(\mathrm{A})$ will denote the closure of the subset $A$ in the space $X$. A space $X$ is said to be $\underline{T}_{1}$ at $\underline{\text { its subset }} \underline{A}$ if each point of $A$ is closed in $X$. $X$ is said to be B-W Compact [1] if every infinite subset of $X$ has at least one limit point. A point $X$ in $X$ is said to be a cluster point ( $w$ - limit point in the terminology of Thron [1]) of a subset $A$ of $X$ if every neighbourhood of $x$ contains an infinite number of points of $A$. $X$ is said to be a Frechet space if whenever $x \in \operatorname{cl}(A)$, there is a sequence of points in $A$ converging to $x$.A map $f: X \rightarrow Y$ is said to be perfect if it is continuous, closed, and has compact fibers $\mathrm{f}^{-1}(\mathrm{y}), \mathrm{y} \in \mathrm{Y}$. For study of perfect maps, see [2] and its references.

The primary purpose of this paper is to give relationships between continuous maps,closed maps, perfect maps, and maps with closed graph. A generalization and an analogue of theorem 5 of Piotrowski and Szymanski [3] and analogues of theorem 1.1.17 and corollary 1.1.18 of Hamlett and Herrington [4] are also obtained.

NOTE. The definitions of subcontinuous and inversely subcontinuous maps can be found in Fuller [5].

\section{MAIN RESULTS.}

THEOREM 1 [4] .Let $\mathrm{f}: \mathrm{X} \rightarrow \mathrm{Y}$ be continuous, where $\mathrm{Y}$ is Hausdorff. Then $\mathrm{f}$ has closed graph.

THEOREM 2. Let $\mathrm{f}: \mathrm{X} \rightarrow \mathrm{Y}$ be closed with closed (compact) fibers, where $\mathrm{X}$ is regular (Hausdorff). Then $\mathrm{f}$ has closed graph.

PROOF. We prove only the parenthesis part; the other part, which can also be proved in a simple manner by using our proof of the parenthesis part, has been proved by Fuller [ 5 ,corollary 3.9]and by Hamlett and Herrington [4, theorem 1.1.17] by different techniques. Let $x \in X, y \in Y, y \neq f(x)$. Then $\mathrm{x} \notin \mathrm{f}^{-1}(\mathrm{y})$, which is compact. Since $\mathrm{X}$ is Hausdorff, there exist disjoint open sets $\mathrm{U}$ and $\mathrm{V}$ containing 
$\mathrm{x}$ and $\mathrm{f}^{-1}(\mathrm{y})$ respectively. Then $\mathrm{f}$ is closed implies there exists an open set $\mathrm{W}$ containing $\mathrm{y}$ such that $\mathrm{f}^{-1}(\mathrm{~W}) \subset \mathrm{V}$ and therefore, $\mathrm{f}(\mathrm{U}) \cap \mathrm{W}=\phi$. It follows that $\mathrm{f}$ has closed graph.

Combining theorems 1 and 2 , we get the following

THEOREM 3. Let $f: X \rightarrow Y$ be perfect, where either $X$ or $Y$ is Hausdorff. Then $f$ has closed graph.

The following theorem 4(theorem 5), part (b) of which is a generalization (analogue) of theorem 5 of Piotrowski and Szymanski [3], gives sufficient conditions under which the converse of theorem 1(theorem 2) holds.

THEOREM 4. Let $\mathrm{f}: \mathrm{X} \rightarrow \mathrm{Y}$ have closed graph. Then $\mathrm{f}$ is continuous if any one of the following conditions is satisfied.

(a) $\mathrm{Y}$ is compact,

(b) $\mathrm{X}$ is Frechet and $\mathrm{Y}$ is $\mathrm{B}-\mathrm{W}$ compact,

(c) $\mathrm{f}$ is subcontinuous.

PROOF. We give the proof of part (b) only; part (a) is well known (corollary 2(b) of Piotrowski and Szymanski [3], and theorem 1.1.10 of [4]), while part (c) is theorem 3.4 of Fuller [5]. Let $F$ be a closed subset of $Y$ and let $x \in \operatorname{clf}^{-1}(F)-f^{-1}(F)$. Since $X$ is a Frechet space, there exists a sequence $\left\{x_{n}\right\}$ of points in $f^{-1}(F)$ such that $x_{n} \rightarrow x$. Since $f$ has closed graph, the set $H$ of values of the sequence $\left\{f\left(x_{n}\right)\right\}$ is an infinite subset of the $B-W$ compact set $F$ and $F$ is $T_{1}$ at $H$. Therefore, $H$ has a cluster point $y \in F$, $y \neq f(x)$, and the set $U=X-f^{-1}(y)$ is an open set containing $x$. Then $x_{n} \rightarrow x$ implies there exists a positive integer $n_{0}$ such that $x_{n} \in U$ for all $n \geq n_{0}$. Again $f$ has closed graph and the set $K=\left\{x_{n}: n \geq n_{0}\right\} U\{x\}$ is compact; it follows that $f(K)$ is closed, which is a contradiction since it is easy to see that $y \in \operatorname{clf}(K)-f(K)$. Hence $f$ must be continuous.

THEOREM. 5. Let $\mathrm{f}: \mathrm{X} \rightarrow \mathrm{Y}$ have closed graph. Then $\mathrm{f}$ is closed if any one of the following conditions is satisfied.

(a) $\mathrm{X}$ is compact,

(b) $\mathrm{X}$ is countably compact and $\mathrm{Y}$ is Frechet,

(c) $\mathrm{f}$ is inversely subcontinuous.

PROOF. We give the proof of part (b) only; part (a) is well known (corollary 2(a) of Piotrowski and Szymanski [3]), while part (c) is theorem 3.5 of Fuller [5].Let $F$ be a closed subset of $X$ and let $y \in \operatorname{clf}(F)-f(F)$. Since $Y$ is Frechet and $T_{1}$ at $f(X)$, there exists a sequence $\left\{f\left(x_{n}\right)\right\}$ of distinct points converging to $y$ where $x_{n} \in F$.Now the set of values of the sequence $\left\{x_{n}\right\}$ is an infinite subset of the countably compact set $F$ and therefore, it has a cluster point $x \in F, y \neq f(x)$. Since $Y$ is $T_{1}$ at $f(X)$, the set $\mathrm{V}=\mathrm{Y}-\{\mathrm{f}(\mathrm{x})\}$ is an open set containing $\mathrm{y}$. Then $\mathrm{f}\left(\mathrm{x}_{\mathrm{n}}\right) \rightarrow \mathrm{y}$ implies there exists a positive integer $\mathrm{n}_{0}$ such that $f\left(x_{n}\right) \in V$ for all $n \geq n_{0}$. Since $f$ has closed graph and the set $K=\left\{f\left(x_{n}\right): n \geq n_{0}\right\} U\{y\}$ is compact, it follows that $f^{-1}(K)$ is closed, which is a contradiction since it is easy to see that $x \in \operatorname{clf}^{-1}(K)-f^{-1}(K)$. Hence $f$ must be closed.

Combining theorems 1 and 5(theorems 2 and 4), we obtain the following theorem 6 (theorem 7), giving a relationship between continuous and closed maps. Theorem 6 includes theorem 16.19 of Thron [1], while theorem 7 includes and gives analogues of corollary 1.1.18 of Hamlett and Herrington [4].

THEOREM 6. Let $f: X \rightarrow Y$ be continuous, where $Y$ is Hausdorff and one of the conditions (a), (b), (c) in theorem 5 is satisfied. Then $f$ is closed.

The condition that $X$ is countably compact in theorems 5(b)and 6(b) cannot be replaced by the weaker condition that $\mathrm{X}$ is $\mathrm{B}-\mathrm{W}$ compact,as the following example shows.

EXAMPLE. Let $X=N$, the positive integers, with a base for a topology on $X$ the family of all sets of the form $\{2 n-1,2 n\}, n \in N$, and $Y=\{0,1,1 / 2, \ldots .1 / n, \ldots\}$ as a subspace of the real line. The map $f: X \rightarrow Y$, defined by $f(2 n-1)=1 / n-1=f(2 n)$ for $n \geq 2$ and $f(1)=0=f(2)$, is a continuous surjection which is not closed, although $\mathrm{X}$ is $\mathrm{B}-\mathrm{W}$ compact and $\mathrm{Y}$ is Frechet, Hausdorff. 
THEOREM 7. Let $f: X \rightarrow Y$ be closed with closed (compact) fibers, where $X$ is regular (Hausdorff) and one of the conditions (a), (b), (c) in theorem 4 is satisfied. Then $f$ is continuous(perfect).

Combining theorems 1 and 4, we obtain the following relationship between continuous maps and maps with closed graph.

THEOREM 8. Let $\mathrm{f}: \mathrm{X} \rightarrow \mathrm{Y}$ be any map, where $\mathrm{Y}$ is Hausdorff and one of the conditions (a), (b), (c) of theorem 4 is satisfied. Then $f$ is continuous if and only if it has closed graph.

Combining theorems 2 and 5 , we obtain the following relationship between closed maps and maps with closed graph.

THEOREM 9. Let $\mathrm{f}: \mathrm{X} \rightarrow \mathrm{Y}$ be any map with closed (compact) fibers, where $\mathrm{X}$ is regular (Hausdorff) and one of the conditions (a), (b), (c) of theorem 5 is satisfied. Then $f$ is closed if and only if it has closed graph.

Combining theorems 3,4 and 5, we obtain the following relationship between perfect maps and maps with closed graph.

THEOREM 10.Let $\mathrm{f}: \mathrm{X} \rightarrow \mathrm{Y}$ be any map with compact fibers, where either $\mathrm{X}$ is Hausdorff or $\mathrm{Y}$ is Hausdorff and one of conditions (a), (b), (c) of theorem 4 and one of the conditions (a), (b), (c) of theorem' 5 are satisfied. Then $\mathrm{f}$ is perfect if and only if it has closed graph.

COROLLARY. Let $\mathrm{f}: \mathrm{X} \rightarrow \mathrm{Y}$ be a bijection and one of the conditions (a), (b), (c) of theorem 4 and one of the conditions (a), (b), (c) of theorem 5 be satisfied.Then $f$ has closed graph if and only if it is a homeomorphism and both $\mathrm{X}, \mathrm{Y}$ are Hausdorff.

Combining theorems 8,9 , and 10 we obtain the following

THEOREM 11. Let $\mathrm{f}: \mathrm{X} \rightarrow \mathrm{Y}$ be any map with closed (compact) fibers, where $\mathrm{X}$ is regular (Hausdorff), $Y$ is Hausdorff, and one of the conditions (a), (b), (c) of theorem 4 and one of the conditions (a), (b), (c) of theorem 5 are satisfied. Then the following conditions (i) to (iii) $\{$ (i) to (iv) $\}$ are equivalent.

(i) $\mathrm{f}$ is continuous.

(ii) $\mathrm{f}$ is closed.

(iii) $f$ has closed graph.

(iv) $\mathrm{f}$ is perfect.

\section{REFERENCES}

1. THRON, W.J. Topological Structures,Holt, Rinehart and Winston, 1966.

2. GARG,G.L.\& GOEL, A. Perfect maps in compact (countably compact) spaces, Int.J.Math And Math. Sci. (To appear).

3. PIOTROWSKI,Z.\& SZYMANSKI, A.Closed graph theorem: topological approach, Rendiconti Del Circolo Matematico Di Palermo Serie II 37(1988), 88-99.

4. HAMLETT, T.R. \& HERRINGTON, L.L. The closed graph and p-closed graph properties in general topology, Amer. Math. Soc. Providence Rhode Island, 1981.

5. FULLER, R.V.Relations among continuous and various non- continuous functions, Pacific J. Math. 25(1968), 495-509. 


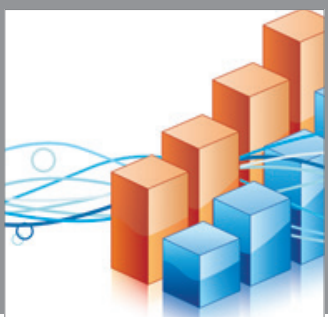

Advances in

Operations Research

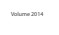

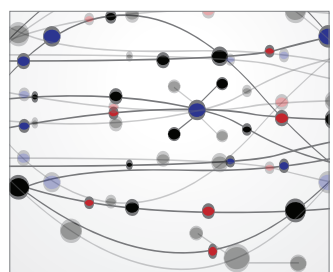

\section{The Scientific} World Journal
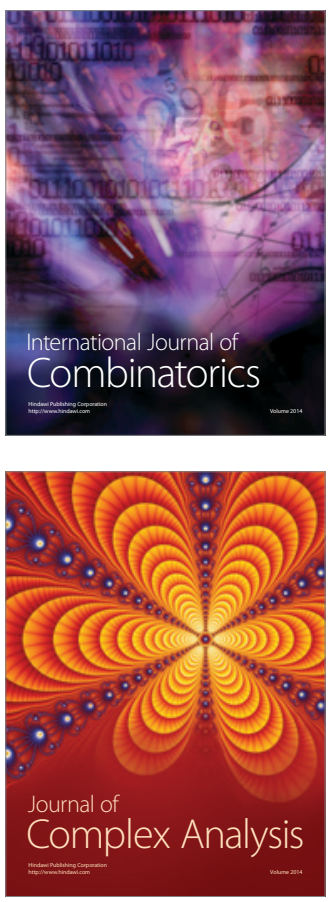

International Journal of

Mathematics and

Mathematical

Sciences
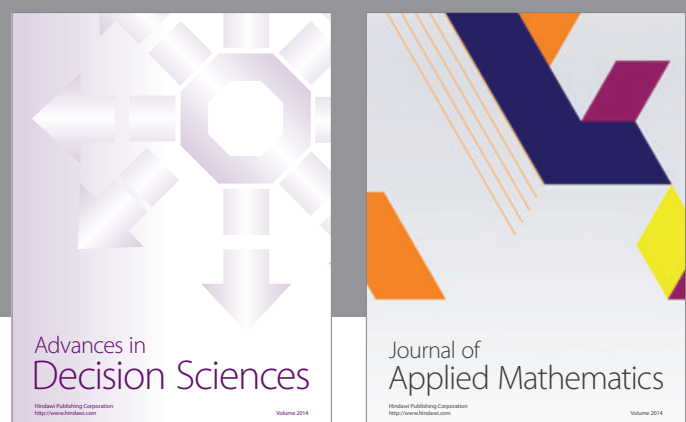

Journal of

Applied Mathematics
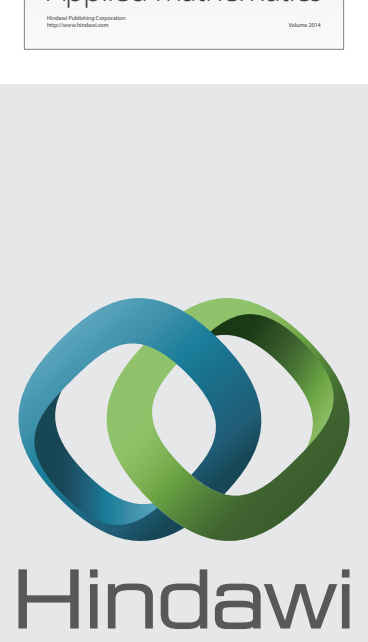

Submit your manuscripts at http://www.hindawi.com
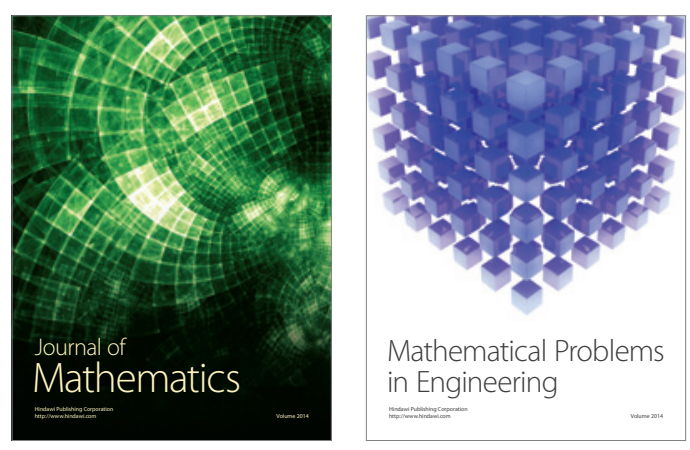

Mathematical Problems in Engineering
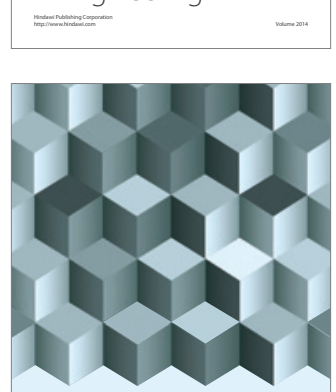

Journal of

Function Spaces
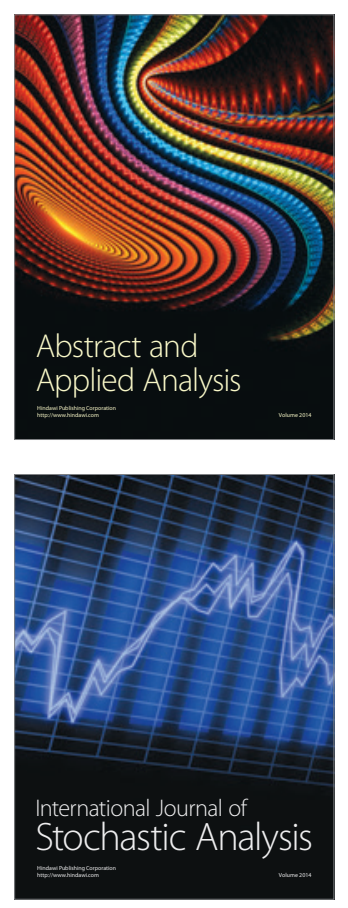

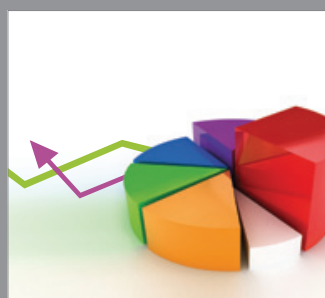

ournal of

Probability and Statistics

Promensencen
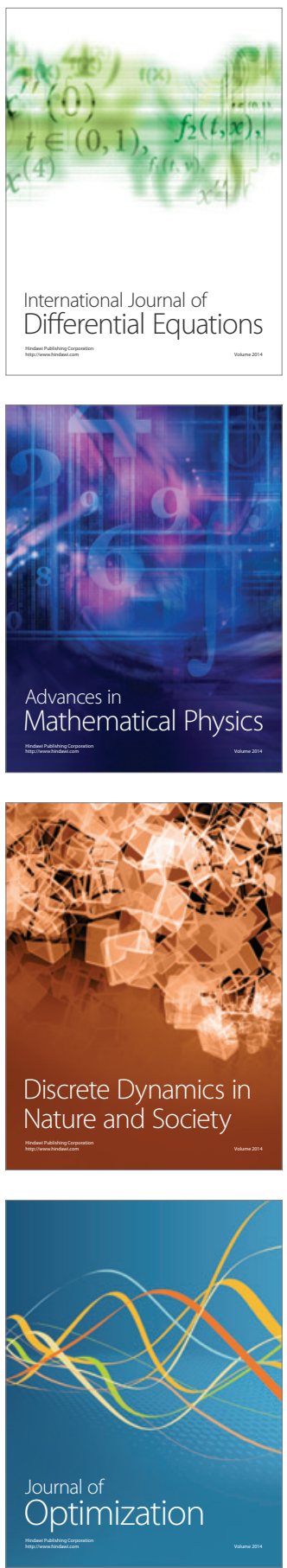